\title{
CPPBA Are Nutritional Composed Scoring Systems Q and Protein-Energy Wasting Score Associated With Mortality in Maintenance Hemodialysis Patients?
}

\author{
Barbara Perez Vogt, MSc, and Jacqueline Costa Teixeira Caramori, PhD
}

Objective: The diagnostic of protein-energy wasting should be done using a tool that can predict clinically important outcomes, besides identifying malnutrition. This study investigated which nutritional composed scoring systems best predicts all-cause mortality in maintenance hemodialysis patients.

Design and Methods: Cohort study that included prevalent patients undergoing hemodialysis for at least 1 month. To assess nutritional status, Subjective Global Assessment (SGA), Malnutrition-Inflammation Score (MIS), and diagnostic criteria for protein-energy wasting proposed by the International Society of Renal Nutrition and Metabolism (ISRNM) were used. Patients were assessed in the moment of inclusion in the study (between July 2012 and December 2012) and followed prospectively to verify the occurrence of deaths.

Results: A total of 163 patients were included, $54.6 \%$ were male, and mean age was $58.4 \pm 15.5$ years. During the follow-up period (15.5 \pm 5.4 months), 29 patients died and 16 underwent kidney transplant. Kaplan-Meier survival curves and Cox proportional hazard analysis adjusted for age, gender, dialysis vintage, diabetes, and serum urea showed that SGA and MIS were predictors of all-cause mortality.

Conclusion: Of the 3 investigated scoring systems, SGA and MIS predict mortality in a period of $15.5 \pm 5.4$ months of follow-up. (c) 2016 Published by Elsevier Inc. on behalf of the National Kidney Foundation, Inc.

This article has an online CPE activity available at www.kidney.org/professionals/CRN/ceuMain.cfm

\section{Introduction}

$\mathrm{P}$ ROTEIN-ENERGY WASTING (PEW) is the state of decreased body stores of protein and energy fuels, which is often associated with increased morbidity and mortality rates in patients undergoing hemodialysis. ${ }^{1}$ In chronic kidney disease (CKD), there are conditions resulting in loss of lean body mass not related solely to decrease in nutrient intake. These include nonspecific inflammatory processes, transient intercurrent catabolic illnesses, nutrient losses into dialysate, loss of blood during dialysis process, acidemia, endocrine disorders, and hyperparathyroidism. ${ }^{1}$

To determine nutritional status, National Kidney Foundation recommends combining measures. ${ }^{2}$ Several simple composed scoring systems have been developed with the purpose of evaluating nutritional status. ${ }^{1,3-5}$ However, there is not a method that can be considered as a gold standard to validate the scoring systems. Moreover,

Faculdade de Medicina de Botucatu, UNESP Univ Estadual Paulista, Department of Clinical Medicine, Botucatu, São Paulo, Brazil.

Financial Disclosure: The authors declare that they have no relevant financial interests.

Address correspondence to Jacqueline Costa Teixeira Caramori, PhD, Faculdade de Medicina de Botucatu, UNESP Univ Estadual Paulista, Distrito de Rubião Junior, s/n , Botucatu, São Paulo, 18618970, Brazil. E-mail: jteixeir@fmb.unesp.br

(C) 2016 Published by Elsevier Inc. on behalf of the National Kidney Foundation, Inc.

$1051-2276 / \$ 36.00$

http://dx.doi.org/10.1053/j.jm.2015.11.003 because they rely on different sets of variables, they result in different nutritional classifications for the same patient. ${ }^{6}$

Subjective Global Assessment (SGA) is a tool initially developed to assess nutritional status in preoperative surgical patients. ${ }^{7}$ It was modified ${ }^{8}$ and validated to screening nutritional risk in CKD patients and showed an association with mortality rates. ${ }^{9,10}$ Malnutrition-Inflammation Score (MIS) was proposed as a nutritional screening for patients undergoing hemodialysis. ${ }^{4}$ Studies have shown its association with morbidity and mortality., ${ }^{41}$ In 2008, the International Society of Renal Nutrition and Metabolism (ISRNM) recommended its diagnostic criteria for $\mathrm{PEW}^{1}$; however, clinical studies using these criteria are still scarce. ${ }^{12-14}$ Currently, ISRNM considers that there is no consensus about the relationship of SGA and MIS to the diagnosis of PEW. These scoring systems should be considered as potential clinical markers of PEW status but not as definitive diagnostic indicators of this syndrome.

A clinically useful nutritional marker should be able to identify the problem, predict clinically important outcomes, such as risk of morbidity and mortality, and be able to identify patients who should receive nutritional intervention and evaluate the response to nutritional interventions. ${ }^{15}$

Therefore, as malnutrition is a significant predictor of mortality in hemodialysis patients, it is important to diagnose PEW with the best available tool to predict outcomes. However, the power of aforementioned 3 scoring systems to predict mortality has not been compared yet. Herein, 
we aimed to evaluate the prevalence of malnutrition, assessed by 3 different nutritional scoring systems and to determine which best predicts all-cause mortality in maintenance hemodialysis patients.

\section{Subjects and Methods Subjects and Study Design}

This retrospective cohort study included prevalent hemodialysis patients of the dialysis of the Hospital of Botucatu Medical School, Universidade Estadual Paulista, Brazil. The patients had been receiving hemodialysis 3 times a week for at least 1 month. Patients with missing data regarding the scoring systems, neoplasia, HIV positivity, and terminal diseases were excluded.

The first nutritional assessment between July 2012 and December 2012 was considered the moment of inclusion in the study. The following demographic and clinical data were obtained from medical records: gender, age, dialysis vintage, diagnosis of diabetes, and cause of end-stage renal disease, biochemical data, and nutritional assessment.

Serum concentrations of urea, creatinine, albumin, total cholesterol, and C-reactive protein were obtained from venous blood samples collected for routine examinations. Blood samples were drawn before a hemodialysis session in a non-fasting state. All measurements were performed at the specialized chemistry laboratory of the Hospital of Botucatu Medical School.

Total iron-binding capacity was calculated from serum values of iron and transferrin. ${ }^{16}$ All patients were monitored and counseled by the nutrition team during the follow-up period, and when necessary, nutritional support was introduced.

\section{Nutritional Status Assessment}

To evaluate nutritional status using SGA, the CANUSAmodified 7-point scale SGA was chosen. ${ }^{8}$ It incorporates 7 components: weight changes, dietary intake, gastrointestinal symptoms, functional capacity, comorbidities, subcutaneous fat, and muscle wasting. The overall score range from 1 (severely malnourished) to 7 (well nourished). Patients were divided into 3 groups, according to the obtained score: A (scores 7 and 6), B (scores 5-3), and C (scores 2 and 1).

MIS incorporates 7 components of the original SGA plus body mass index (BMI), serum albumin level, and total iron-binding capacity or transferrin level. ${ }^{4}$ Each MIS component has 4 levels of severity from 0 (normal) to 3 (very severe). The sum of all 10 components results in an overall score ranging from 0 (normal) to 30 (severely malnourished). The patients were divided into tertiles, according to the obtained sum of the components (first, second, and third tertiles).

To diagnose PEW using an ISRNM-based criteria, the following variables were chosen: biochemical (serum albu$\min <3.8 \mathrm{~g} / \mathrm{dL}$ or cholesterol $<100 \mathrm{mg} / \mathrm{dL}$ ), low body weight, reduced total body fat, or weight loss $($ BMI $<$ $23 \mathrm{~kg} / \mathrm{m}^{2}$, or unintentional weight loss over time [5\% over 3 months or $10 \%$ over 6 months], or total body fat percentage $<10 \%$ ), decrease in muscle mass (mid-arm muscle circumference area $[\mathrm{MAMC}]$ reduction $>10 \%$ in relation to 50 th percentile of reference population) and low protein or energy intakes (protein intake $<0.8 \mathrm{~g} / \mathrm{kg} /$ day and energy intake $<25 \mathrm{kcal} / \mathrm{kg} /$ day). At least 3 of the 4 listed categories (and at least 1 test in each of the selected category) must be satisfied for the diagnosis of kidney disease-related PEW.

Anthropometric assessment was performed after the end of hemodialysis session. Body weight, height, mid-arm circumference, and skinfold thickness (triceps, biceps, suprailiac, and subscapular) were measured. Body weight was measured to the nearest $0.1 \mathrm{~kg}$ with a balance-beam scale, and height was measured with patients standing erect and recorded to the nearest $0.5 \mathrm{~cm}$. Mid-arm circumference was measured using a flexible tape in the halfway point between the acromion of the scapula and the olecranon of the ulna, with the arm relaxed and flexed $90^{\circ}$. Skinfolds thicknesses were measured on the opposite side to the arteriovenous fistula or on the non-dominant side using a Lange skinfold caliper (Cambridge Instrument, Cambridge, Massachusetts), according to standard techniques. ${ }^{17}$ Using these measures, BMI and body fat percentage $e^{18,19}$ were calculated. Percent standard of tricipital skinfold thickness (TST) and MAMC were obtained from the National Health and Nutrition Examination Survey percentile distribution tables adapted by Frisancho. ${ }^{20}$

Dietary intake was evaluated using 3-day food diaries (1 hemodialysis session day and 2 non-dialysis days). Patients were instructed by dietitians to register all foods and the amounts consumed. Energy and protein intake were analyzed using the software NutWin (UNIFESP, São Paulo, São Paulo, Brazil).

\section{Follow-Up and End Points}

The prevalent hemodialysis patients were evaluated between July 2012 and December 2012, and the occurrence of deaths was verified until June 2014. Patients were censored if they were switched off dialysis, underwent renal transplantation, or were transferred to another facility.

\section{Statistical Analysis}

Data were expressed as mean \pm standard deviation, and frequencies were expressed as percentages. Generalized linear model was used to compare survival and nonsurvival groups.

The outcome of interest in the present study was allcause mortality. Kaplan-Meier survival analyses were used to calculate cumulative survival probabilities, and the difference between SGA groups, tertiles of MIS, and ISRNMbased criteria was assessed by the log-rank test. Thereafter, to calculate the relative risks of death, hazard ratios, and $95 \%$ confidence interval were obtained by separate Cox 


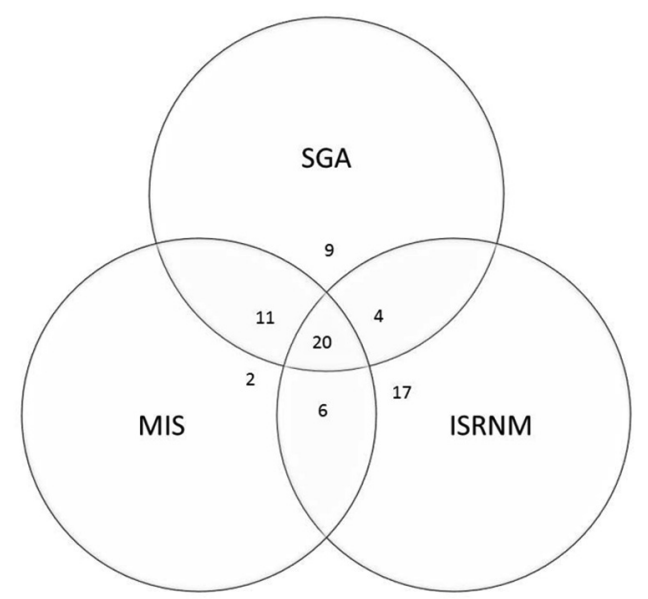

Figure 1. Venn diagram showing malnutrition classifications according to 3 criteria: Subjective Global Assessment (SGA), Malnutrition-Inflammation Score (MIS), and International Society of Renal Nutrition and Metabolism (ISRNM)-based criteria.

proportional hazard models for each scoring system, adjusted for the relevant covariates. Differences were considered statistically significant when $P$ value $<0.05$. Statistical analysis was performed using SPSS 22.0.

\section{Patients}

\section{Results}

A total of 163 patients undergoing maintenance hemodialysis, 3 times weekly for 4 hours, were followed for $15.5 \pm 5.4$ months in this study. The causes of end-stage renal disease were diabetic nephropathy $(29 \%)$, hypertensive nephrosclerosis (18.5\%), undetermined (18.5\%), chronic glomerulonephritis $(9.3 \%)$, polycystic kidney disease $(3.7 \%)$, and others (21\%). Most of the patients were male, and 72 were diabetic.

The prevalence of malnutrition was: $26.3 \%$ by SGA (SGA < 6), $25.2 \%$ by MIS (MIS > 8), and $28.8 \%$ by ISRNM-based criteria. Sixty-nine patients were classified as having malnutrition by at least 1 of the criteria. Only 20 were classified as having malnutrition by the 3 criteria (Fig. 1). Demographic, clinical, and nutritional characteristics of the patients included are summarized in Table 1.

\section{Follow-Up and End Points}

At the end of the study period of $15.5 \pm 5.4$ months, 29 $(17.8 \%)$ patients had died, $16(9.8 \%)$ patients had received kidney transplants, and 118 (72.4\%) patients were still on maintenance hemodialysis. None of the patients had switched off dialysis or had been transferred to another facility.

\section{Comparison Between Survivals and Non- Survivals}

Comparisons of demographic, clinical, and nutritional characteristics of survivals and non-survivals patients are summarized in Table 1. The follow-up time of nonsurvival patients was significantly lower than that of survival patients. Age was significantly higher in the non-survival group; serum urea and albumin of non-survival patients were significantly lower. Malnutrition assessed by SGA and MIS was more prevalent in the non-survival groups, whereas malnutrition assessed by ISRNM-based criteria did not differ between the groups.

\section{Comparison Between Malnourished and Well- Nourished Patients}

Creatinine and albumin serum levels, BMI, and MAMC were significantly lower among patients of group C of SGA (poorest nutritional status) when compared to group A. $\mathrm{BMI}$ and MAMC also were significantly lower when compared to group B. Patients of group B had lower serum creatinine levels and lower BMI than group A.

Patients were analyzed according to tertiles of MIS: first tertile (1-5 points) included 55 patients; second tertile (5-7 points), 54 patients; and third tertile (7-28 points), 54 patients. Patients included in the third tertile of MIS (poorest nutritional status) had significantly lower creatinine and albumin serum levels, BMI, and MAMC when compared to the first one and significantly lower BMI, TST, and MAMC when compared to the second one.

Malnourished patients for ISRNM-based system had significantly lower serum creatinine level and decreased BMI, TST, and MAMC than well-nourished patients.

\section{Kaplan-Meier Survival Analysis}

In Kaplan-Meier survival analysis, patients in SGA group $A$ (scores 7 and 6) showed higher survival rates than those in SGA groups B (scores 3-5; $P<.01$ ) and $\mathrm{C}$ (scores 1 and 2; $P<.01)$. No significant differences between groups $B$ and $\mathrm{C}$ were found $(P=.06$; Fig. $2 \mathrm{~A})$.

Survival rates of MIS tertiles were significantly different (first vs. third tertile $P<.01$; second vs. third tertile $P<.01)$. Survival rates of first tertile did not differ from second one $(P=.78)$. Patients with lowest scores showed higher survival rates than patients with highest scores (Fig. 2B).

No difference in survival rates between well-nourished and malnourished patients according to ISRNM-based criteria were found $(P=.11$; Fig. $2 \mathrm{C})$.

\section{Cox Proportional Hazard Analysis}

Three models were fitted using Cox proportional hazards analysis, each one for one of the scoring systems. Crude Cox proportional hazards analysis for mortality showed that SGA and MIS were significant predictors for mortality (Table 2).

Multivariate Cox proportional hazards analysis showed that SGA and MIS were significant predictors for mortality after adjustments (Table 2). The only significant and independent risk factor for mortality was nutritional status assessed by SGA and MIS. In the multivariate model 1, the 
Table 1. Baseline Demographic, Clinical, and Nutritional Characteristics of 163 Maintenance Hemodialysis Patients and Comparison Between Survivals and Non-Survivals

\begin{tabular}{|c|c|c|c|c|}
\hline Characteristic & All $(n=163)$ & Survivals $(n=134)$ & Non-survivals $(n=29)$ & $P$ \\
\hline Gender (male [\%]) & $89(54.6)$ & $73(54.5)$ & $16(55.2)$ & NS \\
\hline Age (y) & $58.4 \pm 15.5$ & $57.2 \pm 15$ & $64.1 \pm 16.9$ & $<.05$ \\
\hline Presence of diabetes (\%) & $72(44.2)$ & $55(41)$ & $17(58.6)$ & NS \\
\hline Dialysis vintage (mo) & $43.6 \pm 52.9$ & $43.5 \pm 55.1$ & $44.1 \pm 42.3$ & NS \\
\hline Follow-up time (mo) & $15.5 \pm 5.4$ & $17.1 \pm 4$ & $8.2 \pm 5.4$ & $<.01$ \\
\hline Serum urea (mg/dL) & $107.7 \pm 32.9$ & $110.3 \pm 32.7$ & $95.5 \pm 31.8$ & $<.05$ \\
\hline Serum creatinine $(\mathrm{mg} / \mathrm{dL})$ & $8.5 \pm 2.8$ & $8.7 \pm 2.9$ & $7.6 \pm 2.6$ & NS \\
\hline Serum albumin $(g / d L)$ & $3.8 \pm 0.6$ & $3.9 \pm 0.6$ & $3.6 \pm 0.4$ & $<.05$ \\
\hline CRP (mg/dL) & $1.7 \pm 2.6$ & $1.6 \pm 2.8$ & $2.0 \pm 1.5$ & NS \\
\hline $\mathrm{BMI}\left(\mathrm{kg} / \mathrm{m}^{2}\right)$ & $26.1 \pm 6.6$ & $26.2 \pm 6.0$ & $25.6 \pm 8.9$ & NS \\
\hline TST (\%) & $109.6 \pm 65.2$ & $111.4 \pm 67.1$ & $100.7 \pm 55.3$ & NS \\
\hline MAMC (\%) & $98.5 \pm 17.8$ & $99.4 \pm 16.8$ & $94.4 \pm 21.9$ & NS \\
\hline \multicolumn{5}{|l|}{ SGA (n [\%]) } \\
\hline A & $120(73.6)$ & $107(79.3)$ & $13(46.4)$ & $<.01$ \\
\hline $\mathrm{B}$ & $33(20.3)$ & $23(17)$ & $10(35.7)$ & \\
\hline $\mathrm{C}$ & $10(6.1)$ & $5(3.7)$ & 5 (17.9) & \\
\hline \multicolumn{5}{|l|}{ MIS (n [\%]) } \\
\hline$\leq 8$ & $122(74.8)$ & 111 (82.2) & $11(39.3)$ & $<.01$ \\
\hline$>8$ & $41(25.5)$ & $24(17.8)$ & $17(60.7)$ & \\
\hline 1st tertile & $55(33.7)$ & $50(37)$ & $5(17.9)$ & $<.01$ \\
\hline 2nd tertile & $54(33.1)$ & $50(37)$ & 4 (14.3) & \\
\hline 3rd tertile & 54 (33.1) & $35(25.9)$ & 19 (67.9) & \\
\hline \multicolumn{5}{|l|}{ ISRNM (n [\%]) } \\
\hline Yes & 116 (71.2) & $36(26.7)$ & $11(39.3)$ & NS \\
\hline No & 47 (28.8) & 99 (73.3) & $17(60.7)$ & \\
\hline
\end{tabular}

BMI, body mass index; CRP, C-reactive protein; ISRNM, International Society of Renal Nutrition and Metabolism criteria; MAMC, percent standard mid-arm muscle circumference; MIS, Malnutrition-Inflammation Score; SGA, Subjective Global Assessment; NS, not significant; TST, percent standard triciptal skinfold thickness.

decrease in 1 unit of SGA score increases 33\% the risk to mortality, whereas the increase in 1 unit of MIS increases $15 \%$ the risk of mortality. In the ISRNM-based criteria model, none of the variables was a significant and independent risk factor for mortality.

\section{Discussion}

The present study evaluates the impact of different nutritional status scoring systems on mortality of prevalent patients on maintenance hemodialysis. Nutritional status evaluation is very important because poor nutritional status contributes to high morbidity and mortality. ${ }^{1,4}$ It was observed that patients classified as malnourished by SGA and MIS had a higher mortality risk.

Recently, our research group published a study aiming to validate scoring systems in patients undergoing hemodialysis. ${ }^{14}$ Three qualitative criteria to assess nutritional status were compared: criteria by Wolfson et al., ${ }^{5}$ ISRNM, ${ }^{1}$ and Beberashvili et al. $^{3}$ Among these criteria, ISRNM was the best one to predict mortality in 2 years of followup. This accuracy was attributed to the fact that ISRNM criteria consider diverse variables (anthropometric, laboratory, and relating to food intake) and it was the only one to include the component of calorie-protein intake compared to the other scores. ${ }^{14}$
Gracia-Iguacel et al. ${ }^{12}$ verified that ISRNM criteria did not have prognostic value in patients undergoing hemodialysis. The diagnosis of PEW by ISRNM criteria can be done using several combinations. Maybe these possibilities could be a limitation to an accurate diagnosis. The same criteria results in different nutritional classifications for the same patient, depending on the chosen markers. Mazairac et al. ${ }^{13}$ did different combinations of some of the components of ISRNM criteria and verified that the cutoffs of serum albumin, BMI, serum creatinine, and protein catabolic rate recommended by ISRNM had a strong association with mortality. However, serum albumin, or serum creatinine alone was better predictor of outcomes.

Some of the criteria are not reliable measures, and there are limitations due to the nature of CKD, including anthropometric measures, nutrients intake, and biochemical parameters. For example, weight change may be due to fluid shifts. The quantification of energetic and protein intake from food register frequently may be underestimated by CKD patients. ${ }^{21-25}$ Other non-reliable criteria are serum cholesterol and albumin. Serum cholesterol may be affected by medicaments often prescribed to CKD patients. Serum albumin may be affected by inflammation and hydration status of the patient. Despite these limitations, serum albumin has been an important marker in the nutritional status assessment and mortality risk. ${ }^{26,27}$ 

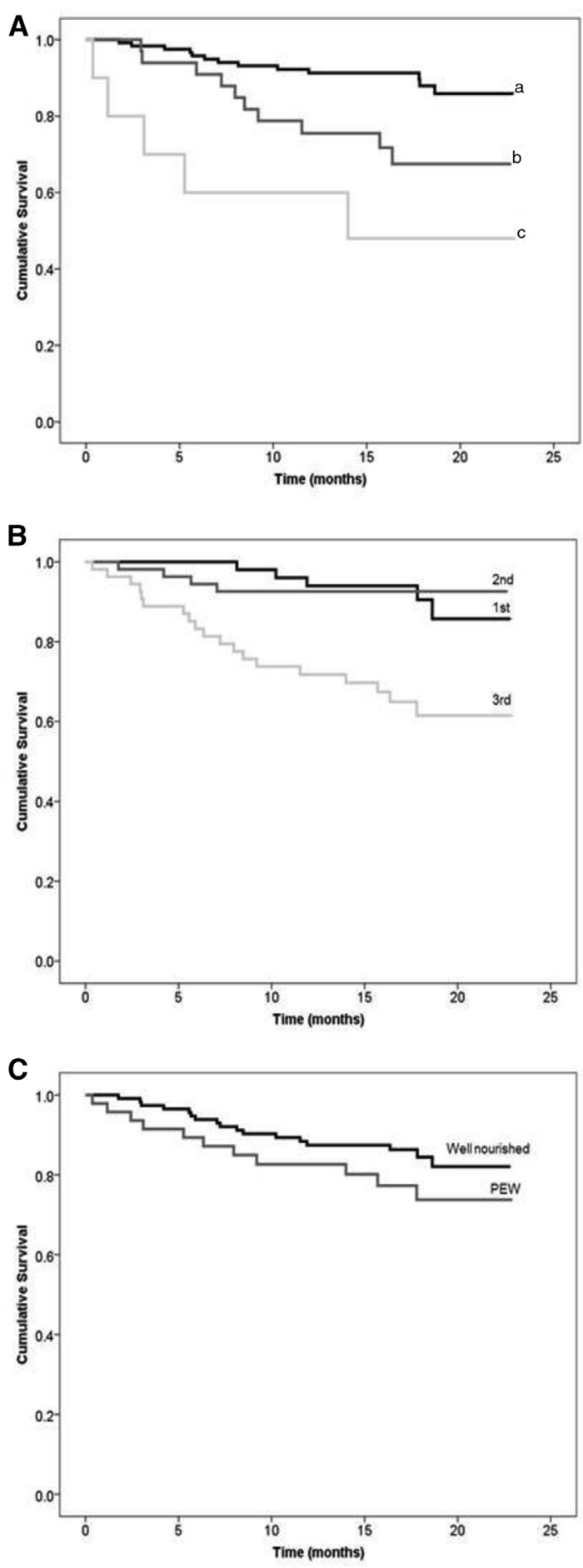

Figure 2. Kaplan-Meier survival analysis of Subjective Global Assessment (A): a (scores 7 and 6), b (scores 5-3), c (scores 2 and 1); Malnutrition-Inflammation Score (B): first (1st), second (2nd), and third (3rd) tertiles; International Society of Renal Nutrition and Metabolism based criteria (C): wellnourished or protein-energy wasting.

Fiedler et al. ${ }^{28}$ assessed malnutrition using SGA, MIS, and nutritional risk screening. All these scoring systems were associated with mortality in 3 years, and the highest hazard ratio to mortality was from MIS. Several studies verified the association of malnutrition with mortality using only 1 of the criteria chosen by us to diagnose malnutrition. ${ }^{10-12,29-31}$ SGA and MIS are well-established criteria and largely used in clinical studies; however, they were compared in few studies.

Other studies tested single nutritional markers to predict mortality. ${ }^{26,27,31,32}$ In the present study, anthropometric parameters did not differ between survivals and nonsurvivals. Only serum urea and albumin were significantly decreased in non-survival group. Serum urea is a marker of protein intake, and serum albumin is a nutritional status marker but is affected by the presence of inflammation. Although many measurements of malnutrition or inflammation correlate with clinical outcome, these values generally do not evaluate clinical condition and outcome in a combined way for an individual patient.

Composed scoring systems, such as SGA, MIS, and ISRNM incorporate objective and subjective aspects in the nutritional status assessment of hemodialysis patients. Therefore, composed scores may be more sensitive and specific methods. Moreover, because they involve these many aspects, it may represent an overall concept of nutritional status. Beberashvili et al. ${ }^{33}$ used MIS to evaluate whether it reflects longitudinal changes in nutritional status. Changes in MIS were associated with changes in energetic and protein intake, serum creatinine, MAMC, interleukin 6, body fat percentage, and free fat mass. Changes in MIS were also associated with increased risk of hospitalization and mortality.

Unfortunately, it was not possible to compare the scoring systems used in this study because some were quantitative and other qualitative. As'habi et al. ${ }^{6}$ compared SGA, MIS, and dialysis malnutrition score and found similar results to identify PEW in patients undergoing hemodialysis. Hou et al. ${ }^{34}$ found a correlation between quantified and modified SGA and MIS.

More recently, other composed score systems were tested in maintenance hemodialysis patients. The geriatric nutritional risk index is a simple and objective tool, which uses only 3 objective parameters of body weight, height, and serum albumin, and has been shown as a good predictor of mortality. ${ }^{25,35,36}$ SGA and MIS include more subjective components than geriatric nutritional risk index. Another simple score based on ISRNM criteria was proposed by a French group, and it was able to predict mortality in patients undergoing hemodialysis. ${ }^{37}$

The limitations of the present study were: (1) inclusion of prevalent patients, and not incident; (2) the sample size was small and consisted of patients from a single center; (3) longitudinal changes in nutritional status were not evaluated during this relatively short time of follow-up. Despite these limitations, it is noteworthy that the results of this study are significant and may base robust studies and guide the choice of the best method to assess nutritional status in patients on maintenance hemodialysis. 
Table 2. Cox Proportional Hazard Analysis for 3 Nutritional Status Scoring Systems Applied to 163 Maintenance Hemodialysis Patients

\begin{tabular}{|c|c|c|c|c|c|c|}
\hline \multirow[b]{2}{*}{ Models } & \multicolumn{2}{|c|}{ SGA } & \multicolumn{2}{|c|}{ MIS } & \multicolumn{2}{|c|}{ ISRNM } \\
\hline & HR (95\% Cl) & $P$ & HR (95\% Cl) & $P$ & HR (95\% Cl) & $P$ \\
\hline Crude & $0.64(0.53-0.77)$ & $<.01$ & $1.15(1.08-1.21)$ & $<.01$ & $0.55(0.26-1.16)$ & .12 \\
\hline Multivariate 1 & $0.67(0.55-0.81)$ & $<.01$ & $1.15(1.08-1.22)$ & $<.01$ & $0.57(0.26-1.26)$ & .17 \\
\hline Multivariate 2 & $0.68(0.55-0.84)$ & $<.01$ & $1.14(1.07-1.22)$ & $<.01$ & $0.63(0.28-1.39)$ & .25 \\
\hline
\end{tabular}

$\mathrm{Cl}$, confidence interval; HR, hazard ratio; ISRNM, International Society of Renal Nutrition and Metabolism criteria; MIS, MalnutritionInflammation Score; SGA, Subjective Global Assessment.

Multivariate model 1 included age, gender, and dialysis vintage as independent variables, in addition to nutritional status evaluated by the scoring systems. Multivariate model 2 included age, gender, dialysis vintage, diabetes, and serum urea as independent variables, in addition to nutritional status evaluated by the scoring systems.

In conclusion, the prevalence of malnutrition was: $26.3 \%$ by SGA (SGA < 6 ), $25.2 \%$ by MIS (MIS > 8 ), and $28.8 \%$ by ISRNM-based criteria. Malnutrition assessed by SGA and MIS was able to predict mortality in a period of $15.5 \pm 5.4$ months of follow-up in this sample of a single center. The set of variables chosen from ISRNM criteria was not able to predict mortality in this sample. Because of the increased risk of mortality in the presence of malnutrition, the nutritional status of dialysis patients should be assessed regularly. Early detection of risk of malnutrition may allow early-initiating specific nutritional strategies, to prevent more accentuated nutritional status deterioration.

\section{Practical Application}

A major issue in determining malnutrition or proteinenergy wasting in maintenance hemodialysis patients is the lack of a gold standard. Nutritional composed scoring systems have been used to diagnose malnutrition and to be validated, they should predict clinical outcomes. The results of the present study add evidence to the question of which nutritional score should be used on clinical practice. Malnutrition Inflammation Score and Subjective Global Assessment predict the outcome mortality in this cohort of hemodialysis patients. Early detection of malnutrition using those tools may allow early initiating specific nutritional strategies, which can prevent nutritional status deterioration and poor outcomes.

\section{References}

1. Fouque D, Kalantar-Zadeh K, Kopple J, et al. A proposed nomenclature and diagnostic criteria for protein-energy wasting in acute and chronic kidney disease. Kidney Int. 2008;73:391-398.

2. Clinical practice guidelines for nutrition in chronic renal failure. K/ DOQI, National Kidney Foundation. Am J Kidney Dis. 2000;35:S1-S140.

3. Beberashvili I, Azar A, Sinuani I, et al. Objective Score of Nutrition on Dialysis (OSND) as an alternative for the malnutrition-inflammation score in assessment of nutritional risk of haemodialysis patients. Nephrol Dial Transplant. 2010;25:2662-2671.

4. Kalantar-Zadeh K, Kopple JD, Block G, Humphreys MH. A malnutrition-inflammation score is correlated with morbidity and mortality in maintenance hemodialysis patients. Am J Kidney Dis. 2001;38:1251-1263.
5. Wolfson M, Strong CJ, Minturn D, Gray DK, Kopple JD. Nutritional status and lymphocyte function in maintenance hemodialysis patients. Am J Clin Nutr. 1984;39:547-555.

6. As'habi A, Tabibi H, Nozary-Heshmati B, Mahdavi-Mazdeh M, Hedayati M. Comparison of various scoring methods for the diagnosis of protein-energy wasting in hemodialysis patients. Int Urol Nephrol. 2014;46:999-1004.

7. Detsky AS, McLaughlin JR, Baker JP, et al. What is subjective global assessment of nutritional status? J Parenter Enteral Nutr. 1987;11:8-13.

8. Adequacy of dialysis and nutrition in continuous peritoneal dialysis: association with clinical outcomes. Canada-USA (CANUSA) Peritoneal Dialysis Study Group. J Am Soc Nephrol. 1996;7:198-207.

9. Steiber A, Leon JB, Secker D, et al. Multicenter study of the validity and reliability of subjective global assessment in the hemodialysis population.J Ren Nutr. 2007;17:336-342.

10. Stenvinkel P, Barany P, Chung SH, Lindholm B, Heimburger O. A comparative analysis of nutritional parameters as predictors of outcome in male and female ESRD patients. Nephrol Dial Transplant. 2002;17:1266-1274.

11. Rambod M, Bross R, Zitterkoph J, et al. Association of MalnutritionInflammation Score with quality of life and mortality in hemodialysis patients: a 5-year prospective cohort study. Am J Kidney Dis. 2009;53:298-309.

12. Gracia-Iguacel C, Gonzalez-Parra E, Perez-Gomez MV, et al. Prevalence of protein-energy wasting syndrome and its association with mortality in haemodialysis patients in a centre in Spain. Nefrologia. 2013;33:495-505.

13. Mazairac AH, de Wit GA, Grooteman MP, et al. A composite score of protein-energy nutritional status predicts mortality in haemodialysis patients no better than its individual components. Nephrol Dial Transplant. 2011;26:1962-1967.

14. Toledo FR, Antunes AA, Vannini FC, et al. Validity of malnutrition scores for predicting mortality in chronic hemodialysis patients. Int Urol Nephrol. 2013;45:1747-1752.

15. Jeejeebhoy KN. Nutritional assessment. Gastroenterol Clin North Am. 1998;27:347-369.

16. Kalantar-Zadeh K, Kleiner M, Dunne E, et al. Total iron-binding capacity-estimated transferrin correlates with the nutritional subjective global assessment in hemodialysis patients. Am J Kidney Dis. 1998;31:263-272.

17. Heyward VH, Stolarczyk LM. Dobras cutâneas. In: Avaliação da composição corporal aplicada [Applied body composition assessment]. Barueri: Manole; 2000: p. 23-46.

18. Durnin JV, Womersley J. Body fat assessed from total body density and its estimation from skinfold thickness: measurements on 481 men and women aged from 16 to 72 years. Br J Nutr. 1974;32:77-97.

19. Siri WE. Body composition from fluid spaces and density: analysis of methods. 1961. Nutrition. 1993;9:480-491.

20. Frisancho AR. New norms of upper limb fat and muscle areas for assessment of nutritional status. Am J Clin Nutr. 1981;34:2540-2545.

21. Bazanelli AP, Kamimura MA, Vasselai P, Draibe SA, Cuppari L. Underreporting of energy intake in peritoneal dialysis patients. $J$ Ren Nutr. 2010;20:263-269. 
22. Fassett RG, Robertson IK, Geraghty DP, Ball MJ, Coombes JS. Dietary intake of patients with chronic kidney disease entering the LORD trial: adjusting for underreporting. J Ren Nutr. 2007;17:235-242.

23. Kloppenburg WD, de Jong PE, Huisman RM. The contradiction of stable body mass despite low reported dietary energy intake in chronic haemodialysis patients. Nephrol Dial Transplant. 2002;17:1628-1633.

24. Mafra D, Moraes C, Leal VO, Farage NE, Stockler-Pinto MB, Fouque D. Underreporting of energy intake in maintenance hemodialysis patients: a cross-sectional study. J Ren Nutr. 2012;22:578-583.

25. Kobayashi I, Ishimura E, Kato Y, et al. Geriatric Nutritional Risk Index, a simplified nutritional screening index, is a significant predictor of mortality in chronic dialysis patients. Nephrol Dial Transplant. 2010;25:3361-3365.

26. Antunes AA, Delatim Vannini F, de Arruda Silveira LV, Martin LC, Barretti P, Caramori JC. Influence of protein intake and muscle mass on survival in chronic dialysis patients. Ren Fail. 2010;32:1055-1059.

27. Araujo IC, Kamimura MA, Draibe SA, et al. Nutritional parameters and mortality in incident hemodialysis patients. J Ren Nutr. 2006;16:27-35.

28. Fiedler R, Jehle PM, Osten B, Dorligschaw O, Girndt M. Clinical nutrition scores are superior for the prognosis of haemodialysis patients compared to lab markers and bioelectrical impedance. Nephrol Dial Transplant. 2009;24:3812-3817.

29. de Mutsert R, Grootendorst DC, Boeschoten EW, et al. Subjective global assessment of nutritional status is strongly associated with mortality in chronic dialysis patients. Am J Clin Nutr. 2009;89:787-793.
30. Segall L, Mardare NG, Ungureanu S, et al. Nutritional status evaluation and survival in haemodialysis patients in one centre from Romania. Nephrol Dial Transplant. 2009;24:2536-2540.

31. Segall L, Moscalu M, Hogas S, et al. Protein-energy wasting, as well as overweight and obesity, is a long-term risk factor for mortality in chronic hemodialysis patients. Int Urol Nephrol. 2014;46:615-621.

32. Chan M, Kelly J, Batterham M, Tapsell L. Malnutrition (subjective global assessment) scores and serum albumin levels, but not body mass index values, at initiation of dialysis are independent predictors of mortality: a 10year clinical cohort study. J Ren Nutr. 2012;22:547-557.

33. Beberashvili I, Azar A, Sinuani I, et al. Comparison analysis of nutritional scores for serial monitoring of nutritional status in hemodialysis patients. Clin J Am Soc Nephrol. 2013;8:443-451.

34. Hou Y, Li X, Hong D, et al. Comparison of different assessments for evaluating malnutrition in Chinese patients with end-stage renal disease with maintenance hemodialysis. Nutr Res. 2012;32:266-271.

35. Park JH, Kim SB, Shin HS, Jung YS, Rim H. Geriatric nutritional risk index may be a significant predictor of mortality in Korean hemodialysis patients: a single center study. Ther Apher Dial. 2012;16:121-126.

36. Panichi V, Cupisti A, Rosati A, et al. Geriatric nutritional risk index is a strong predictor of mortality in hemodialysis patients: data from the Riscavid cohort. J Nephrol. 2014;27:193-201.

37. Moreau-Gaudry X, Jean G, Genet L, et al. A simple protein-energy wasting score predicts survival in maintenance hemodialysis patients. J Ren Nutr. 2014;24:395-400. 